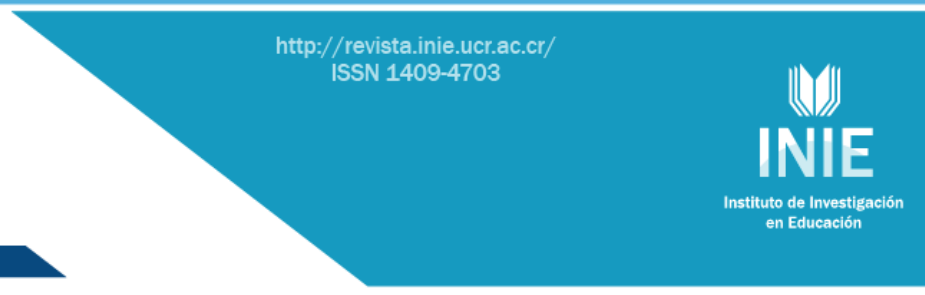

\title{
ANÁLISIS COMPARATIVO DE CRITERIOS DE DESEMPEÑO PROFESIONAL PARA LA ENSEÑANZA EN CUATRO PAÍSES DE AMERICA \\ COMPARATIVE ANALYSIS OF PROFESSIONAL PERFORMANCE CRITERIA FOR TEACHING IN FOUR COUNTRIES OF AMERICA
}

\section{Volumen 14, Número 3 \\ Setiembre - Diciembre}

pp. 1-20

Este número se publicó el 30 de setiembre de 2014

María del Ángel Vázquez Cruz

Graciela Cordero Arroyo

Yolanda E. Leyva Barajas

Revista indizada en $\underline{\text { REDALYC, SCIELO }}$

Revista distribuida en las bases de datos:

CATÁLOGO DE LATINDEX, IRESIE, CLASE, DIALNET, DOAJ, E-REVIST@S, SHERPA/ROMEO, QUALIS, MIAR

Revista registrada en los directorios:

ULRICH'S, REDIE, RINACE, OEI, MAESTROTECA, PREAL, $\underline{\text { CLACSO }}$ 


\title{
ANÁLISIS COMPARATIVO DE CRITERIOS DE DESEMPEÑO PROFESIONAL PARA LA ENSEÑANZA EN CUATRO PAÍSES DE AMERICA \\ COMPARATIVE ANALYSIS OF PROFESSIONAL PERFORMANCE CRITERIA FOR TEACHING IN FOUR COUNTRIES OF AMERICA
}

\author{
María del Ángel Vázquez Cruz ${ }^{1}$ \\ Graciela Cordero Arroyo ${ }^{2}$ \\ Yolanda E. Leyva Barajas ${ }^{3}$
}

\begin{abstract}
Resumen: Una de las tendencias de los sistemas educativos para fortalecer la profesión docente ha sido la elaboración de marcos para la enseñanza o criterios de desempeño profesional, que sirven como base para la formación inicial y continua, la evaluación de la práctica y la definición de la identidad docente. Al respecto, diversos países han establecido sus marcos a partir del consenso entre docentes, investigadores y autoridades educativas. Por lo anterior, el presente ensayo tiene como finalidad revisar los modelos para definir criterios de desempeño profesional propuestos en cuatro países: los Estados Unidos, Chile, Perú y México. Los modelos se analizaron en forma comparativa en tres ejes: finalidad, componentes y dominios. A partir de esta revisión, el ensayo propone algunas orientaciones que podrían retomarse para la elaboración de marcos para la enseñanza en otros contextos.
\end{abstract}

Palabras clave: ESTÁNDARES DE DESEMPEÑO, COMPETENCIAS DOCENTES, DESARROLLO PROFESIONAL, EVALUACIÓN DOCENTE, ESTADOS UNIDOS, CHILE, PERÚ, MÉXICO.

\begin{abstract}
One of the trends of education systems to strengthen the teaching profession has been to develop frameworks for teaching or professional performance criteria. They serve as a basis for initial and continuing training, assessment practice and the definition of teacher identity. Several countries have established their frames to be discussed by teachers, researchers and educational authorities. This paper aims to review the frameworks for good teaching developed in four countries: the United States, Chile, Peru and Mexico. The models were analyzed comparatively in three areas: purpose, components and domains. From this review, the paper proposes some guidelines which could be taken up for the development of frameworks for teaching in other contexts.
\end{abstract}

Key words: PERFORMANCE STANDARS, TEACHER QUALIFICATIONS, PROFESSIONAL DEVELOPMENT, PERFORMANCE ASSESSMENT, UNITED STATES OF AMERICA, CHILE, PERU, MEXICO.

\footnotetext{
1 Estudiante de la Maestría en Ciencias Educativas del Instituto de Investigación y Desarrollo Educativo de la Universidad Autónoma de Baja California (UABC), México. (Posgrado de Calidad Consolidado CONACYT). Licenciada en Ciencias de la Educación por la UABC. Dirección electrónica: vc.mariangel@gmail.com

2 Investigadora Titular del Instituto de Investigación y Desarrollo Educativo de la Universidad Autónoma de Baja California (UABC), México. Miembro del Sistema Nacional de Investigadores desde 1999. Licenciada en pedagogía por la Universidad Nacional Autónoma de México. Doctora en Filosofía y Ciencias de la Educación por la Universidad de Barcelona. Dirección electrónica: gcordero@uabc.edu.mx

${ }^{3}$ Miembro de la Red Iberoamericana de Investigadores sobre Evaluación de la Docencia y asesora de la presidencia del Instituto Nacional para la Evaluación de la Educación (INEE), México. Licenciada en Psicología y Maestra en Análisis Experimental de la Conducta por la UNAM. Doctora en Educación por la Universidad de Aguascalientes (Padrón de Excelencia CONACYT). Dirección electrónica: yleyva@inee.edu.mx
}

Ensayo recibido: 20 de enero, 2014

Devuelto para corrección: 2 de junio, 2014

Aprobado: 18 de agosto, 2014 


\section{Introducción}

Las mayores demandas y exigencias sociales sobre la calidad de la educación han aumentado las expectativas sobre el desempeño de los profesores de Educación Básica. De hecho, las políticas docentes de los países de América Latina han sido tema de análisis durante los primeros años de este siglo para organismos internacionales como la Organización para la Cooperación y el Desarrollo Económico (OCDE) y la Oficina Regional de Educación para América Latina y el Caribe (Orealc) de la Organización de las Naciones Unidas para la Educación, la Ciencia y la Tecnología (Unesco, por sus siglas en ingles).

De acuerdo con la OCDE (2010), una de las reformas más importantes que puede implementar un sistema educativo para mejorar la calidad en los centros escolares es el establecimiento de una política integral para seleccionar, preparar, desarrollar y evaluar a los docentes. Para lograrlo se requiere definir estándares que precisen los conocimientos, habilidades y valores relacionados con la buena enseñanza. Dichos referentes serán la base que articule las estrategias para atraer mejores candidatos docentes, fortalecer la formación inicial docente, mejorar la evaluación inicial docente, someter las plazas docentes a concurso, crear períodos de inducción y prueba, mejorar el desarrollo profesional y evaluar para ayudar a mejorar.

Por su parte, la OREALC/UNESCO (2013), en el documento Antecedentes y Criterios para la Elaboración de Políticas Docentes en América Latina y el Caribe, propuso algunas orientaciones para la elaboración de las políticas relativas a la profesión docente, a partir de una revisión de la situación de los docentes y las políticas públicas de los países de la región relacionadas con: (a) formación inicial, (b) formación y desarrollo profesional continuo, y (c) carrera docente. De acuerdo con el documento, las políticas docentes deben tener una posición central y estratégica en los diseños de las políticas educativas, de manera que sean abordadas desde una perspectiva integral y sistémica, por lo que es fundamental contar con una definición válida y consensuada de la buena enseñanza y estándares de desempeño docente.

En este sentido, la conceptualización del buen desempeño docente, a partir de marcos para la enseñanza ${ }^{4}$ o criterios de desempeño profesional, se entiende como una primera etapa para definir programas de formación de profesores y desarrollar sistemas

\footnotetext{
4 Los marcos para la buena enseñanza definen criterios de desempeño profesional docente a partir de competencias o estándares.
} 
de evaluación del desempeño profesional (OCDE, 2010b). Según Vaillant (2004) esta ha sido una de las acciones prioritarias de las políticas relacionadas con el fortalecimiento de la profesión docente y el desarrollo profesional en países como los Estados Unidos, Canadá, Reino Unido, Australia, Escocia y Francia.

En América Latina, Chile y Perú son dos de los países que cuentan con un marco para la enseñanza oficial. En el caso de México, durante el año 2010 se desarrolló un documento para discusión de los estándares de desempeño docente en el aula para los maestros de Educación Básica; sin embargo, la propuesta no fue reconocida por la Secretaría de Educación Pública (SEP) (Barrera y Myers, 2011). En ausencia de estándares docentes y marco para la buena enseñanza oficial en México, Schmelkes y Mancera (OCDE, 2010b) recomiendan revisar la experiencia internacional en la materia. Enfatizan que "el punto de partida para la evaluación docente es describir con precisión los elementos de un buen desempeño de enseñanza" (p. 5).

Dado que la elaboración de marcos para la enseñanza o criterios de desempeño profesional para docentes es una de las tendencias para mejorar la calidad de la profesión a nivel internacional (Feldman y laies, 2010; Vaillant, 2004), el objetivo de este artículo es revisar tres marcos para la enseñanza: uno de los Estados Unidos, dos latinoamericanos (Chile y Perú), y el documento elaborado en México para la discusión de los estándares docentes. La revisión de estos documentos tiene la finalidad de comparar y proporcionar orientaciones que se pueden retomar para un ejercicio de discusión en un contexto nacional o local.

\section{Consideraciones sobre los marcos para la buena enseñanza}

Para Vaillant (2004), los marcos para la enseñanza representan los conocimientos, capacidades, roles y responsabilidades que se consideran necesarios para el desempeño de la labor docente en el aula y la comunidad. Estos criterios identifican, de manera general, las principales características de una buena enseñanza. Un marco puede ser útil como referente para la evaluación de programas de formación, inicial y continua; desarrollar procesos de evaluación, autoevaluación y supervisión del desempeño docente; y la acreditación, selección y promoción durante la carrera docente.

La evaluación puede clasificarse de acuerdo a la finalidad en: (a) sumativa, (b) formativa y (c) mixta (Jornet, Leyva, y Sánchez, 2008). Las evaluaciones con fines sumativos se orientan al control y rendición de cuentas. La información que aportan sirve 
para tomar decisiones acerca de la acreditación, la aprobación, la promoción, el incremento salarial y otras decisiones de alto impacto en la profesión docente (Barrera y Myers, 2011; Jornet et al., 2008). Los procesos de evaluación que tienen propósitos formativos buscan identificar los elementos de la práctica que pueden ser objeto de mejora, este tipo de procesos se utiliza para realizar diagnósticos de desempeño, elaborar trayectos formativos, identificar necesidades de asesoría, facilitar la autoevaluación y promover la reflexión, entre otras actividades actividades que se consideran de bajo impacto, debido a que no ponen en juego la carrera profesional de los docentes (Barrera y Myers, 2011; Jornet et al., 2008). Las evaluaciones que con una finalidad mixta tienen propósitos tanto formativos como sumativos.

No existe un consenso acerca de la estructura y los conceptos a partir de los cuales se definen los marcos para la enseñanza. Los términos que se utilizan varían de una propuesta a otra. Feldman y laies (2010) y Meckes (2013) reconocen que los marcos para la enseñanza se definen principalmente a partir de dos enfoques, el de las competencias o estándares ${ }^{5}$.

El término competencias tiene múltiples definiciones dado que se ha establecido a partir de la influencia de diversas disciplinas y tendencias (Tobón, 2006). En el ámbito educativo se consideran "procesos complejos de desempeño con idoneidad en un determinado contexto, con responsabilidad" (Tobón, 2006, p. 6). Según Camero (2008) la evaluación de las competencias requiere: (1) definir la situación a evaluar, es decir, el perfil profesional que precisa las competencias; (2) especificar los propósitos de la evaluación; (3) establecer los referentes evaluativos, lo que implica identificar los indicadores que permitan inferir el dominio de la competencia y establecer los criterios y estándares para realizar el juicio valorativo del grado o nivel en que la domina, y (4) precisar los instrumentos y procedimientos para obtener la información.

Estándar ha sido un concepto ampliamente discutido en el campo educativo por su origen en el sector industrial (Barrera y Myers, 2011). En educación, el término tiene dos acepciones, por un lado, se concibe como la definición de lo que debe saber y hacer un profesional en determinado contexto y, por otro, se define como un insumo para indicar la distancia entre el desempeño del evaluado y el nivel necesario para ser considerado competente (Meckes, 2013). Desde este enfoque, Ingvarson y Kleinhenz (2006)

5 Otros autores no los consideran dos enfoques distintos. Por ejemplo, Jornet, González, Suárez y Perales (2011) plantean que puede definirse un perfil mediante competencias y desglosarse en un sistema de estándares de desempeño para su evaluación. 
identificaron tres componentes de un sistema de evaluación de profesores: (a) estándares de contenido que definan los conocimientos, habilidades y valores que debe tener un profesor para la buena enseñanza, (b) instrumentos de evaluación, es decir, pautas para recabar información acerca del cumplimiento del estándar, y (c) estándares de desempeño, que describan las características que dan cuenta del nivel de dominio de cada estándar.

Desde la perspectiva de Feldman y laies (2010), los objetivos del marco definen si se elabora a partir de competencias o estándares. Cuando la estrategia tiene como fin orientar procesos de formación docente, gestión y mejora educativa, los marcos se definen a partir de competencias; mientras que las propuestas que tienen como enfoque la evaluación de la práctica, se precisan a partir de estándares. Ambos paradigmas están estrechamente relacionados, dado que las competencias se utilizan como referentes para los procesos de evaluación de la práctica docente y, a su vez, los estándares sirven como marco para la planeación de la formación puesto que precisan lo que se espera de un buen profesor (Feldman y laies, 2010).

Para Meckes (2013), los conceptos competencias y estándares son similares dado que ambos precisan referentes consensuados para el desempeño de un profesional dentro de un marco de calidad. De acuerdo con la autora, en los países anglófonos, como los Estados Unidos, Inglaterra, Escocia, Australia y Nueva Zelanda, es común que los sistemas o instituciones educativas desarrollen estrategias a partir de estándares; mientras que en Francia, Canadá y algunos países de América Latina, la tendencia ha sido el desarrollo de marcos o criterios profesionales para la docencia basados en competencias.

\section{Marcos de la Buena Enseñanza en América}

A continuación se describen cuatro modelos para definir los marcos para la enseñanza o criterios de desempeño de los profesionales de la educación en los Estados Unidos (Danielson, 2013b), Chile (Chile, Gobierno de Chile, 2011), Perú (Perú, Ministerio de Educación, 2012) y México (México, Secretaría de Educación Pública, 2010).

\subsection{Los Estados Unidos: Marco para la Enseñanza}

El Marco para la Enseñanza (ME), elaborado por Danielson (2013b) a partir de estudios empíricos e investigaciones teóricas, precisa las responsabilidades de un 
maestro que mejoran el aprendizaje en los estudiantes. El ME define lo que los maestros requieren saber y ser capaces de hacer en su práctica profesional para una enseñanza eficaz. El documento se encuentra alineado con los estándares formulados por el Interstate Teacher Assessment and Support Consortium (INTASC) y se fundamenta en el enfoque constructivista de la enseñanza y el aprendizaje (Danielson, 2013a). Puede ser utilizado para varios propósitos; por ejemplo, como fundamento de una escuela o distrito, para el acompañamiento, el desarrollo profesional y la evaluación docente.

EI ME se estructura en dominios, componentes, elementos e indicadores. Danielson (2013b) define un dominio como ámbitos de responsabilidad de la enseñanza, cada dominio se define a partir de una serie de componentes, los cuales se precisan a partir de los elementos e indicadores. Además, se describen cuatro posibles niveles de desempeño profesional para cada componente, de manera que el profesor puede ubicarse de acuerdo a su desempeño en el nivel insatisfactorio, básico, competente o distinguido. En el Gráfico 1 se muestra la estructura del marco propuesto por Danielson (2013b).

\section{Gráfico 1}

Componentes del Marco para la Enseñanza de Danielson (2013b).

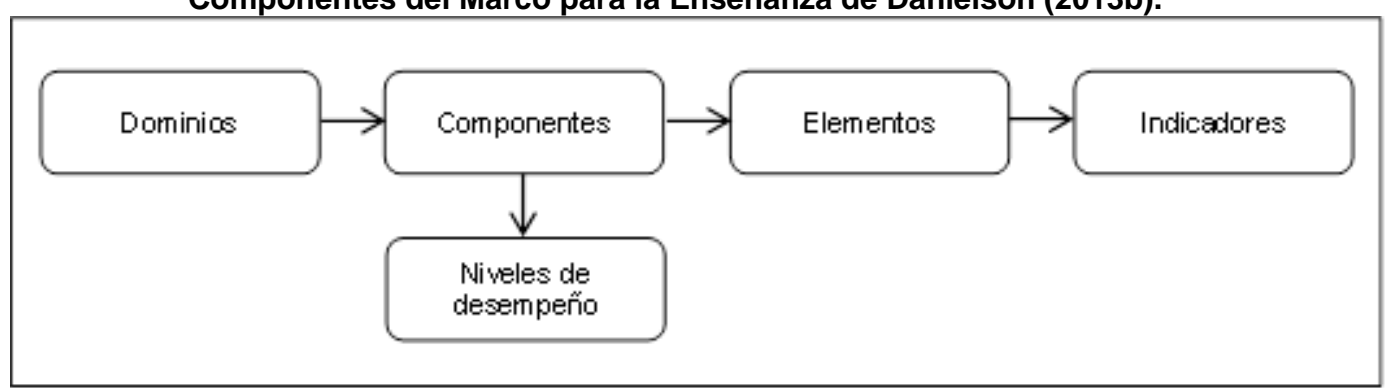

Fuente: Elaboración propia a partir de Danielson (2013b).

En concreto, el ME se estructura en cuatro dominios que agrupan 22 componentes, 76 elementos y una serie de indicadores para cada componente. En la Tabla 1 se presentan los dominios y los componentes en los que se divide la actividad docente en el marco propuesto por Danielson (2013a). 
Tabla 1

Dominios del Marco para la Enseñanza y sus componentes

\begin{tabular}{|c|c|}
\hline Dominios & Componentes \\
\hline Planificación y preparación & $\begin{array}{l}\text { - Demostrar conocimiento del contenido disciplinar y pedagógico } \\
\text { - Demostrar conocimiento de los estudiantes } \\
\text { - Establecer resultados académicos } \\
\text { - Demostrar conocimientos de los recursos } \\
\text { - Proyectos instrucción coherentes } \\
\text { - Diseñar evaluaciones de los estudiantes }\end{array}$ \\
\hline Entorno en el aula & $\begin{array}{l}\text { - La creación de un ambiente de respeto y confianza } \\
\text { - Establecimiento de una cultura de aprendizaje } \\
\text { - Manejo de los procedimientos de aula } \\
\text { - Manejo de la conducta estudiantil } \\
\text { - Organizar el espacio físico }\end{array}$ \\
\hline Instrucción & $\begin{array}{l}\text { - La comunicación con los estudiantes } \\
\text { - Usar técnicas de preguntas y discusión } \\
\text { - Involucrar a los estudiantes en el aprendizaje } \\
\text { - Utilizar evaluaciones en la instrucción } \\
\text { - Demostrar flexibilidad y capacidad de respuesta }\end{array}$ \\
\hline Responsabilidades profesionales & $\begin{array}{l}\text { - Reflexionar sobre la enseñanza } \\
\text { - Mantenimiento de expedientes } \\
\text { - Comunicación con las familias } \\
\text { - Participar en una comunidad profesional } \\
\text { - Crecer y desarrollarse profesionalmente } \\
\text { - Mostrar profesionalidad }\end{array}$ \\
\hline
\end{tabular}

Fuente: Danielson (2013b), The framework, párr. 2-5)

Los componentes que integran el marco establecen los conocimientos, habilidades y actitudes que debe tener el docente durante la planeación, el desarrollo y la conclusión de una clase. Además, define sus responsabilidades profesionales fuera del aula, como crecer, desarrollarse y participar en una comunidad profesional.

\subsection{Chile: Marco para la Buena Enseñanza}

El Marco para la Buena Enseñanza (MBE) (Chile, Gobierno de Chile, 2011) desarrollado en Chile toma como base el trabajo realizado por Danielson en los Estados Unidos. El marco será utilizado para orientar las políticas de fortalecimiento de la profesión docente, el desarrollo de programas para la formación inicial y el desarrollo profesional y llevar a cabo procesos de evaluación del desempeño. Asimismo, se resalta la importancia del MEB como instrumento para que los docentes examinen sus prácticas, de manera individual o colectiva, a partir de referentes consensuados para el perfeccionamiento y la mejora. 
EI MBE se estructura en dominios, criterios y descriptores. En el marco (Chile, Gobierno de Chile, 2011) se precisa que los dominios hacen referencia a un aspecto distinto de la enseñanza, siguiendo el ciclo total del proceso educativo. Por su parte, los criterios muestran los elementos específicos en los que deben centrarse los profesores. El tercer componente son los descriptores; no obstante, esta publicación no plantea una definición de dicho concepto. Para cada descriptor se precisan cuatro niveles de desempeño: insatisfactorio, básico, competente y destacado. Los niveles de desempeño que gradúan la descripción de la función docente orientan al docente acerca de lo que se espera de él, y permite a los profesores realizar una autoevaluación de su práctica a partir de criterios establecidos en un marco común; se consideran una herramienta para la evaluación y supervisión con finalidad formativa, ya que precisan información cualitativa para la discusión y proponen ámbitos para el crecimiento profesional. En el Gráfico 2 se presenta la estructura del marco.

Gráfico 2

Componentes del Marco para la Buena Enseñanza

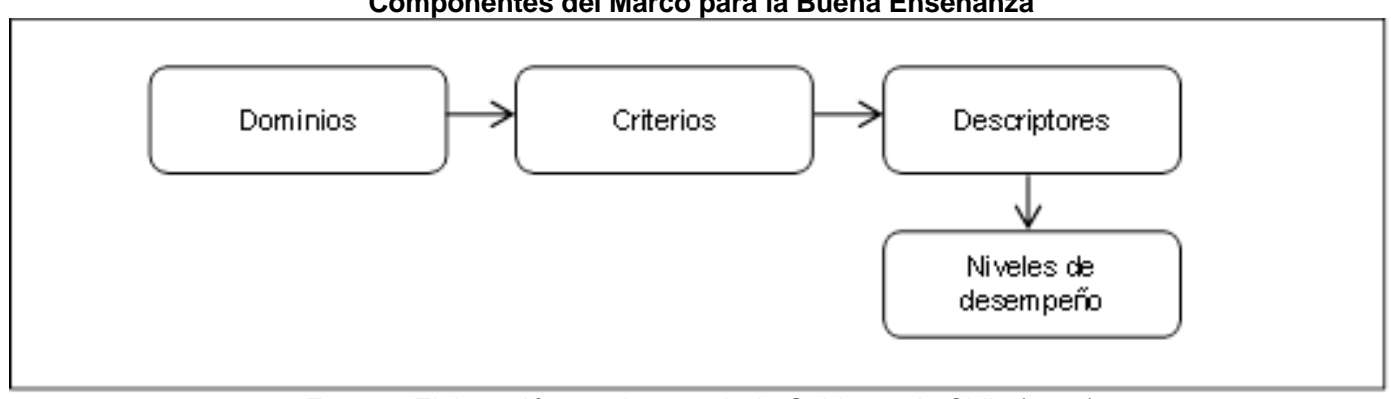

Fuente: Elaboración propia a partir de Gobierno de Chile (2011)

En particular, el marco cuenta con cuatro dominios, los cuales se dividen en 20 criterios y 70 descriptores, como se muestra en la Tabla 2. 
Tabla 2

Dominios del Marco para la Enseñanza y sus componentes

\begin{tabular}{|c|c|}
\hline Dominios & Criterios \\
\hline \multirow{5}{*}{$\begin{array}{l}\text { Preparación de la } \\
\text { Enseñanza }\end{array}$} & A1. Domina los contenidos de las disciplinas que enseña y el marco curricular nacional. \\
\hline & A2. Conoce las características, conocimientos y experiencias de sus estudiantes. \\
\hline & A3. Domina la didáctica de las disciplinas que enseña. \\
\hline & $\begin{array}{l}\text { A4. Organiza los objetivos y contenidos de manera coherente con el marco curricular y las } \\
\text { particularidades de sus alumnos. }\end{array}$ \\
\hline & $\begin{array}{l}\text { A5. Las estrategias de evaluación son coherentes con los objetivos de aprendizaje, la disciplina que } \\
\text { enseña, el marco curricular nacional y permite a todos los alumnos demostrar lo aprendido. }\end{array}$ \\
\hline \multirow{4}{*}{$\begin{array}{c}\text { Creación de un } \\
\text { ambiente propicio } \\
\text { para el aprendizaje }\end{array}$} & B1. Establece un clima de relaciones de aceptación, equidad, confianza, solidaridad y respeto. \\
\hline & $\begin{array}{l}\text { B2. Manifiesta altas expectativas sobre las posibilidades de aprendizaje y desarrollo de todos sus } \\
\text { alumnos. }\end{array}$ \\
\hline & B3. Establece y mantiene normas consistentes de convivencia en el aula. \\
\hline & $\begin{array}{l}\text { B4. Establece un ambiente organizado de trabajo y dispone los espacios y recursos en función de } \\
\text { los aprendizajes. }\end{array}$ \\
\hline \multirow{6}{*}{$\begin{array}{c}\text { Enseñanza para el } \\
\text { aprendizaje de } \\
\text { todos los } \\
\text { estudiantes }\end{array}$} & C1. Comunica en forma clara y precisa los objetivos de aprendizaje. \\
\hline & C2. Las estrategias de enseñanza son desafiantes, coherentes y significativas para los estudiantes. \\
\hline & $\begin{array}{l}\text { C3. El contenido de la clase es tratado con rigurosidad conceptual y es comprensible para los } \\
\text { estudiantes. }\end{array}$ \\
\hline & C4. Optimiza el tiempo disponible para la enseñanza. \\
\hline & C5. Promueve el desarrollo del pensamiento. \\
\hline & $\begin{array}{l}\text { C6. Evalúa y monitorea el proceso de comprensión y apropiación de los contenidos por parte de los } \\
\text { estudiantes. }\end{array}$ \\
\hline \multirow{5}{*}{$\begin{array}{l}\text { Responsabilidades } \\
\text { profesionales }\end{array}$} & D1. El profesor reflexiona sistemáticamente sobre su práctica. \\
\hline & D2. Construye relaciones profesionales y de equipo con sus colegas. \\
\hline & D3. Asume responsabilidades en la orientación de sus alumnos. \\
\hline & D4. Propicia relaciones de colaboración y respeto con los padres y apoderados. \\
\hline & D5. Maneja información actualizada sobre su profesión, el sistema educativo y las políti \\
\hline
\end{tabular}

Fuente: Chile, Gobierno de Chile (2011, p.11)

Al igual que el marco estadounidense (Danielson, 2013b), el MBE parte de los conocimientos, habilidades y valores que se esperan del docente en la preparación, desarrollo y conclusión de una clase, y define las responsabilidades que tiene el profesional fuera del aula. 


\subsection{Perú: Marco de Buen Desempeño Docente}

El Marco de Buen Desempeño Docente (MBDD) (Perú, Ministerio de Educación, 2012) establecido en Perú fue elaborado con base al trabajo realizado en Chile. El documento define los dominios, competencias y desempeños que determinan la buena práctica de los docentes de Educación Básica de este país. El marco fue construido a partir de un acuerdo entre el Estado, los profesores y la sociedad con los siguientes propósitos (Perú, Ministerio de Educación, 2012, p. 17):

- Establecer un lenguaje común entre los que ejercen la profesión docente y los ciudadanos para referirse a los distintos procesos de la enseñanza.

- Promover que los docentes reflexionen sobre su práctica, se apropien de los desempeños que caracterizan la profesión y construyan, en comunidades de práctica, una visión compartida de la enseñanza.

- Promover la revaloración social y profesional de los docentes, para fortalecer su imagen como profesionales competentes que aprenden, se desarrollan y se perfeccionan en la práctica de la enseñanza.

- Guiar y dar coherencia al diseño e implementación de políticas de formación, evaluación, reconocimiento profesional y mejora de las condiciones de trabajo docente.

EI MBDD (Perú, Ministerio de Educación, 2012) se estructura en un orden jerárquico de tres categorías: dominios, competencias y desempeños. Un dominio se refiere a un ámbito de la práctica docente donde se agrupa una serie de desempeños profesionales que se asocian con el aprendizaje de los estudiantes. Las competencias representan un conjunto de características que se atribuyen al sujeto que actúa en un ámbito determinado; los componentes de una competencia son los recursos, movilizados, contexto, finalidad, eficacia e idoneidad. Los desempeños se definen como las actuaciones observables de la persona que pueden ser descritas y evaluadas y que expresan su competencia, se relaciona con el logro de aprendizajes esperados y la ejecución de tareas asignadas. En la especificación de los desempeños se presentan tres condiciones: (a) una actuación observable, (b) una responsabilidad, es decir, una función, y (c) el resultado esperado. En el Gráfico 3 se presentan los componentes del marco. 


\section{Gráfico 3 \\ Componentes del Marco de Buen Desempeño Docente}

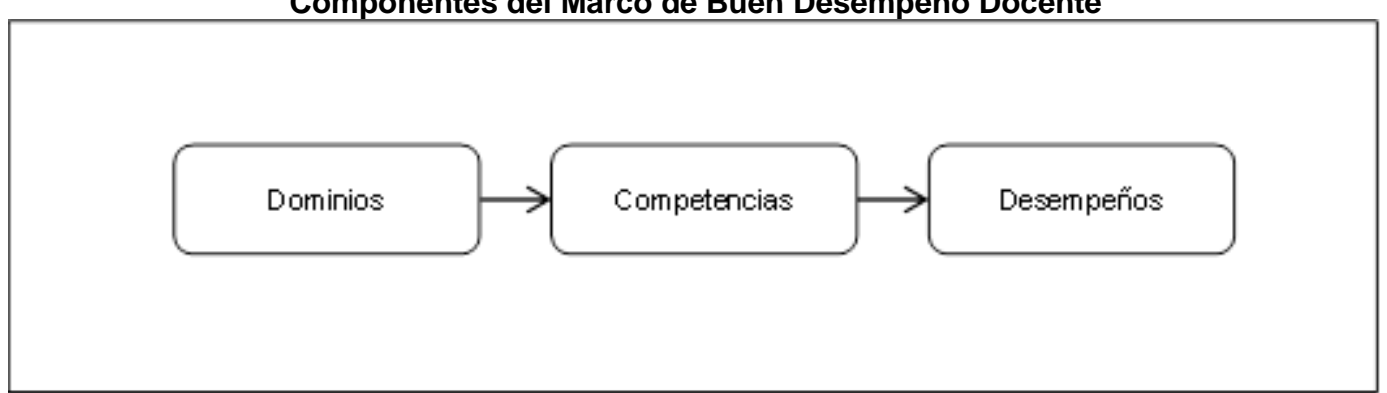

Fuente: Elaboración propia a partir de Perú, Ministerio de Educación (2012).

\section{En la Tabla 3 se presentan los dominios, competencias y el número de desempeños que comprenden la estructura del MBDD.}

Tabla 3

Dominios del Marco para la Enseñanza y sus componentes

\begin{tabular}{|c|c|}
\hline Dominios & Competencias \\
\hline \multirow{2}{*}{$\begin{array}{l}\text { Preparación para el } \\
\text { aprendizaje de los } \\
\text { estudiantes }\end{array}$} & $\begin{array}{l}\text { - Conoce y comprende las características de todos sus estudiantes y sus contextos, los } \\
\text { contenidos disciplinares que enseña, los enfoques y procesos pedagógicos, con el propósito de } \\
\text { promover capacidades de alto nivel y su formación integral. }\end{array}$ \\
\hline & $\begin{array}{l}\text { - Planifica la enseñanza de forma colegiada garantizando la coherencia entre los aprendizajes } \\
\text { que quiere lograr en sus estudiantes, el proceso pedagógico, el uso de los recursos disponibles } \\
\text { y la evaluación, en una programación curricular en permanente revisión. }\end{array}$ \\
\hline \multirow{3}{*}{$\begin{array}{l}\text { Enseñanza para el } \\
\text { aprendizaje de los } \\
\text { estudiantes }\end{array}$} & $\begin{array}{l}\text { - Crea un clima propicio para el aprendizaje, la convivencia democrática y la vivencia de la } \\
\text { diversidad en todas sus expresiones, con miras a formar ciudadanos críticos e interculturales. }\end{array}$ \\
\hline & $\begin{array}{l}\text { - Conduce el proceso de enseñanza con dominio de los contenidos disciplinares y el uso de } \\
\text { estrategias y recursos pertinentes, para que todos los estudiantes aprendan de manera reflexiva } \\
\text { y crítica lo que concierne a la solución de problemas relacionados con sus experiencias, } \\
\text { intereses y contextos culturales. }\end{array}$ \\
\hline & $\begin{array}{l}\text { - Evalúa permanentemente el aprendizaje de acuerdo con los objetivos institucionales previstos, } \\
\text { para tomar decisiones y retroalimentar a sus estudiantes y a la comunidad educativa, teniendo } \\
\text { en cuenta las diferencias individuales y los contextos culturales. }\end{array}$ \\
\hline \multirow{2}{*}{$\begin{array}{c}\text { Participación en la } \\
\text { gestión de la escuela } \\
\text { articulada a la } \\
\text { comunidad }\end{array}$} & $\begin{array}{l}\text { - Participa activamente, con actitud democrática, crítica y colaborativa, en la gestión de la } \\
\text { escuela, contribuyendo a la construcción y mejora continua del Proyecto Educativo Institucional } \\
\text { y así pueda generar aprendizajes de calidad. }\end{array}$ \\
\hline & $\begin{array}{l}\text { - Establece relaciones de respeto, colaboración y corresponsabilidad con las familias, la } \\
\text { comunidad y otras instituciones del Estado y la sociedad civil; aprovecha sus saberes y recursos } \\
\text { en los procesos educativos y da cuenta de los resultados. }\end{array}$ \\
\hline \multirow{2}{*}{$\begin{array}{l}\text { Desarrollo de la } \\
\text { profesionalidad y la } \\
\text { identidad docente }\end{array}$} & $\begin{array}{l}\text { - Reflexiona sobre su práctica y experiencia institucional y desarrolla procesos de aprendizaje } \\
\text { continuo de modo individual y colectivo, para construir y armar su identidad y responsabilidad } \\
\text { profesional. }\end{array}$ \\
\hline & $\begin{array}{l}\text { - Ejerce su profesión desde una ética de respeto de los derechos fundamentales de las personas, } \\
\text { demostrando honestidad, justicia, responsabilidad y compromiso con su función social. }\end{array}$ \\
\hline
\end{tabular}

Fuente: Perú, Ministerio de Educación (2012, pp. 22-23) 
Los primeros dos dominios del marco de Perú se centran en las competencias relacionadas con la preparación y el desarrollo de la enseñanza para el aprendizaje de los estudiantes. Los últimos dos dominios tienen que ver con competencias del docente fuera del aula.

\subsection{México: Estándares de Desempeño Docente en el Aula para la Educación Básica}

En el marco de la Reforma Integral de la Educación Básica (RIEB), en México se publica para su discusión el documento Estándares de Desempeño Docente en el Aula para la Educación Básica (México, Secretaría de Educación Pública, 2010). El documento refiere que la finalidad central de estos estándares es contribuir al desarrollo de juicios evaluativos que favorezcan la mejora continua de la práctica docente en el aula por medio de procesos de evaluación reflexiva y colaborativa.

Los Estándares de Desempeño Docente en México (México, Secretaría de Educación Pública, 2010) se organizan en cinco categorías con sus respectivos referentes de los cuales se desprenden los estándares de desempeño, los niveles de desempeño y las pautas para observarlos. Las categorías corresponden al nivel más general de descripción y a partir de los referentes constituyen las acciones centrales y comunes que realizan los profesores en su práctica en el aula. Mientras que los estándares y niveles de desempeño muestran diversas posibilidades de cómo lo maestros llevan a la práctica cada una de las acciones. Por último, se precisan las pautas para observar, describen elementos en los que puede enfocarse el evaluador para determinar el nivel de desempeño de los docentes en cada estándar. En el Gráfico 4 se observa la organización de los componentes de este documento.

Gráfico 4

Componentes de los Estándares de Desempeño Docente en el Aula para la Educación Básica

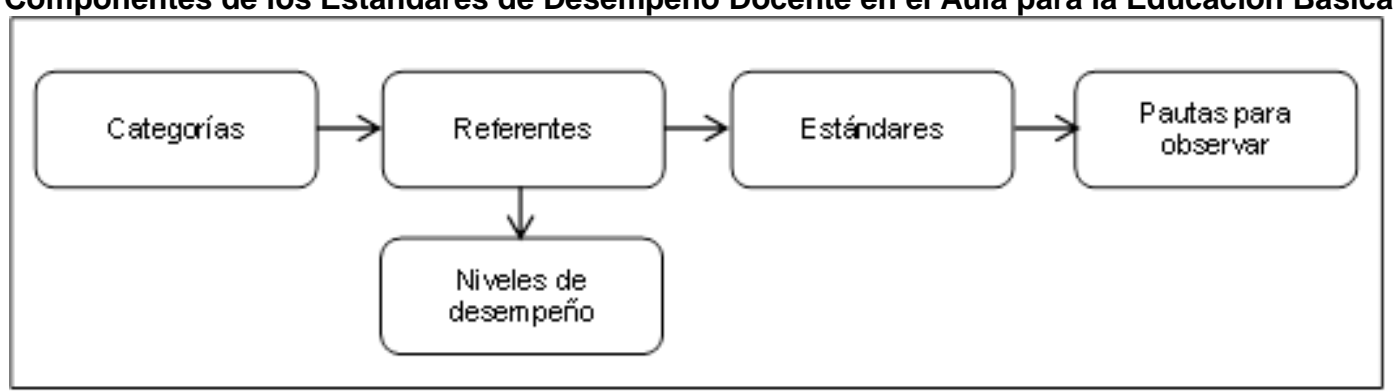

Fuente: Elaboración propia a partir de México, Secretaría de Educación Pública (2010) 
En la Tabla 4 se presentan las categorías de los estándares de desempeño, su definición y los referentes que corresponden a cada una.

Tabla 4

Categorías y referentes de los Estándares de Desempeño Docente en el Aula para la Educación Básica

\begin{tabular}{|c|c|c|}
\hline Categorías & Definición & Referentes \\
\hline Planeación & $\begin{array}{l}\text { Preparación previa que hace el docente del qué, } \\
\text { cómo y para qué de la clase, con el objetivo de } \\
\text { propiciar el aprendizaje de los alumnos. }\end{array}$ & $\begin{array}{l}\text { - Selección de contenidos } \\
\text { - Selección de propósitos } \\
\text { - Siseño de estrategias didácticas } \\
\text { - Selección de mecanismos de evaluación }\end{array}$ \\
\hline $\begin{array}{c}\text { Gestión del } \\
\text { ambiente de la clase }\end{array}$ & $\begin{array}{l}\text { Construcción de un clima propicio para el } \\
\text { aprendizaje. }\end{array}$ & $\begin{array}{l}\text { - Relaciones interpersonales } \\
\text { - Manejo de grupo }\end{array}$ \\
\hline Gestión curricular & $\begin{array}{l}\text { Conocimiento y puesta en práctica del conjunto } \\
\text { de saberes que integran los contenidos de las } \\
\text { asignaturas. }\end{array}$ & $\begin{array}{l}\text { - Conocimiento de las asignaturas } \\
\text { - } \text { Relaciones entre asignaturas } \\
\text { - Conexión asignaturas-contextos }\end{array}$ \\
\hline Gestión didáctica & $\begin{array}{l}\text { Conocimiento y puesta en práctica del conjunto } \\
\text { de saberes (conocimientos) y acciones } \\
\text { metodológicas (uso de métodos y estrategias) } \\
\text { orientadas a promover procesos de aprendizaje } \\
\text { en los alumnos. }\end{array}$ & $\begin{array}{l}\text { - } \text { Presentación curricular } \\
\text { - } \text { Actividades diferenciadas } \\
\text { - } \text { Relación de aprendizaje alumno-alumno } \\
\text { - Recursos didácticos } \\
\text { - } \text { Recursos espaciales } \\
\text { - } \text { Indicaciones } \\
\text { - } \text { Explicaciones } \\
\text { - } \\
\text { - } \text { Actividades dirigidas } \\
\text { - } \text { Actividades no dirigidas }\end{array}$ \\
\hline Evaluación & $\begin{array}{l}\text { Acciones que realizan docentes y alumnos con } \\
\text { el fin de expresar valoraciones, mediante la } \\
\text { sistematización de evidencias pertinentes sobre } \\
\text { los procesos y los resultados del aprendizaje de } \\
\text { los alumnos. }\end{array}$ & $\begin{array}{l}\text { - } \text { Autoevaluación } \\
\text { - Valoración entre los alumnos } \\
\text { - Raloración del docente a los alumnos } \\
\text { - Retroalimentación de saberes }\end{array}$ \\
\hline
\end{tabular}

Fuente: México, Secretaría de Educación Pública (2010, pp. 28-41)

Para evaluar los estándares se establecieron cuatro niveles de desempeño para cada referente, como se observa en la Tabla 5. El nivel cuatro es el estándar de desempeño deseable (México, Secretaría de Educación Pública, 2010). 
Tabla 5

Niveles de desempeño de los Estándares de Desempeño Docente en el Aula para la Educación Básica

\begin{tabular}{|c|l|}
\hline Niveles & \multicolumn{1}{|c|}{ Descripción } \\
\hline Nivel 1 & $\begin{array}{l}\text { Indica que su desempeño es elemental y, por lo tanto, el proceso de mejora implica un trabajo más } \\
\text { intenso, de tal manera que paulatinamente se acerque a los niveles posteriores. }\end{array}$ \\
\hline Nivel 2 & $\begin{array}{l}\text { Hace alusión a una práctica que se distancia de lo sugerido en el estándar, pero que en sí misma se } \\
\text { convierte en una oportunidad de mejora. }\end{array}$ \\
\hline Nivel 3 & $\begin{array}{l}\text { Significa que el docente ha logrado desempeñarse cercanamente a lo señalado en el estándar, pero } \\
\text { que aún puede mejorar. }\end{array}$ \\
\hline Nivel 4 & $\begin{array}{l}\text { Indica una práctica que se desarrolla conforme a lo descrito en el estándar. Se puede considerar una } \\
\text { fortaleza y, por ello, se sugiere sea compartida con el colectivo docente. }\end{array}$ \\
\hline
\end{tabular}

Fuente: México, Secretaría de Educación Pública (2010, p. 27)

\section{Análisis de los cuatro marcos para la enseñanza}

A fin de identificar las semejanzas y diferencias de estos marcos, se revisaron los siguientes elementos: finalidad de los marcos, componentes y dominios de los marcos.

\subsection{Finalidad}

A partir de los objetivos y componentes en los que se organiza la estructura de las propuestas revisadas se identificó que existe una perspectiva predominante en cada uno de los marcos de desempeño. Los marcos desarrollados en los Estados Unidos, Chile y México constituyen propuestas más desarrolladas para llevar a cabo procesos de evaluación, dado que establecen niveles de desempeño que permiten identificar el grado de dominio de los componentes, descriptores y referentes, respectivamente. En los tres casos se propone que la evaluación del desempeño docente sea formativa; sin embargo, pueden utilizarse como referentes para realizar evaluaciones con fines sumativos y consecuencias de alto imparto.

Por ejemplo, en el caso de Chile, se aplica la evaluación "Docentemás" basada en el MEB (Chile, Gobierno de Chile, 2011). Esta evaluación tiene propósitos sumativos y de alto impacto en la carrera docente, es decir, los resultados del proceso llegan a tener consecuencias administrativas, como el acceso a incentivos salariales o la obligación de abandonar la carrera docente cuando no se logran los estándares establecidos (Manzi, González y Sun, 2011).

El marco de Perú está enfocado, principalmente, a objetivos relacionados con la formación de las capacidades necesarias para la enseñanza. En este marco no se 
establecen niveles de desempeño que permitan realizar una evaluación del desempeño docente; no obstante, se espera que sirva de guía para el desarrollo de políticas de evaluación docente (Perú, Ministerio de Educación, 2012).

\subsection{Componentes}

En los marcos de desempeño se utilizan diferentes conceptos para referirse a los componentes que integran el marco en función del nivel de descripción. En el análisis de los marcos se identificaron hasta cinco niveles de descripción que van de lo general (nivel 1) a lo específico (niveles 4 y 5). Los términos utilizados varían de acuerdo al enfoque, la finalidad del marco y el contexto educativo de cada país. En la Tabla 6 se precisan los conceptos utilizados en la estructura de los marcos de desempeño profesional consultados en el presente artículo.

Tabla 6

Conceptos que se utilizan para identificar los niveles de descripción

\begin{tabular}{|c|c|c|c|c|c|}
\hline País & Nivel 1 & Nivel 2 & Nivel 3 & Nivel 4 & Nivel 5 \\
\hline Estados Unidos & Dominios & Componentes & Elementos & $\begin{array}{c}\text { Niveles de } \\
\text { desempeño }\end{array}$ & $\begin{array}{c}\text { Niveles de } \\
\text { desempeño }\end{array}$ \\
\hline Chile & Dominios & Criterios & Descriptores & & Niveles de \\
\hline Perú & Dominios & Competencias & Desempeños & desempeño \\
\hline México & Categorías & Referentes & Estándares & $\begin{array}{c}\text { Pautas para } \\
\text { observar }\end{array}$ & \multicolumn{2}{|c|}{ Fuente: Elaboración propia } \\
\hline
\end{tabular}

Fuente: Elaboración propia

Existen algunas similitudes en los términos utilizados en los niveles de categorización de marcos del desempeño. En particular, los Estados Unidos, Chile y Perú coinciden en que la categoría de descripción más general se denomina dominios. En los tres países donde se tiene como objetivo explícito la evaluación, los Estados Unidos, Chile y México, se establece como último elemento del marco los niveles de desempeño.

Como se mencionó anteriormente, en la literatura se reconoce que los marcos son elaborados a partir de estándares o competencias (Meckes, 2013). De los cuatro modelos revisados se observa que únicamente el marco de Perú se define por medio de competencias y solo la propuesta realizada en México incluye el concepto estándares. Los modelos de los Estados Unidos y Chile utilizan los términos de referentes y componentes, respectivamente. Al contrastar las características de estos marcos con los elementos de una propuesta basada en estándares (Ingvarson y Kleinhenz, 2006) se encontró que ambas cuentan con los tres componentes, es decir, definen estándares de 
contenido, pautas para evaluar y estándares de desempeño; sin embargo, no utilizan el concepto estándares.

\subsection{Dominios de los marcos para la enseñanza}

A partir de los cuatro dominios del Marco para la Enseñanza de los Estados Unidos se categorizaron los niveles más generales de descripción de los marcos de desempeño, dominios en el caso de Chile y Perú, y categorías en los estándares de México. Los dominios se ubicaron en una categoría de acuerdo a su contenido. En la Tabla 7 se presentan los resultados de dicha categorización.

Tabla 7

Dominios de los marcos de los marcos para la enseñanza o criterios de desempeño profesional docente

\begin{tabular}{|c|c|c|c|}
\hline Estados Unidos & Chile & Perú & México \\
\hline \multirow{2}{*}{$\begin{array}{l}\text { Planificación y } \\
\text { preparación }\end{array}$} & \multirow{2}{*}{$\begin{array}{l}\text { Preparación de la } \\
\text { enseñanza }\end{array}$} & \multirow{2}{*}{$\begin{array}{l}\text { Preparación para el } \\
\text { aprendizaje de los } \\
\text { estudiantes }\end{array}$} & Planeación \\
\hline & & & Gestión curricular \\
\hline Entorno en el aula & $\begin{array}{l}\text { Creación de un ambiente } \\
\text { propicio para el aprendizaje }\end{array}$ & \multirow{3}{*}{$\begin{array}{l}\text { Enseñanza para el } \\
\text { aprendizaje de los } \\
\text { estudiantes }\end{array}$} & $\begin{array}{c}\text { Gestión del ambiente de la } \\
\text { clase }\end{array}$ \\
\hline \multirow{2}{*}{ Instrucción } & \multirow{2}{*}{$\begin{array}{c}\text { Enseñanza para el } \\
\text { aprendizaje de todos los } \\
\text { estudiantes }\end{array}$} & & Gestión didáctica \\
\hline & & & Evaluación \\
\hline \multirow{2}{*}{$\begin{array}{l}\text { Responsabilidades } \\
\text { profesionales }\end{array}$} & & $\begin{array}{c}\text { Participación en la gestión } \\
\text { de la escuela articulada a la } \\
\text { comunidad }\end{array}$ & \\
\hline & $\begin{array}{l}\text { Responsabilidades } \\
\text { profesionales }\end{array}$ & $\begin{array}{l}\text { Desarrollo de la } \\
\text { profesionalidad y la } \\
\text { identidad docente }\end{array}$ & \\
\hline
\end{tabular}

Fuente: Elaboración propia

En México solo se precisan los estándares docentes dentro del aula, por lo que no se establecen referentes respecto a las responsabilidades profesionales de los docentes. En los marcos de los Estados Unidos (Danielson, 2013b), Chile (Chile, Gobierno de Chile, 2011) y Perú (Perú, Ministerio de Educación, 2012), este dominio implica actividades relacionadas con la reflexión de la práctica; las relaciones entre los profesores y los miembros de la comunidad educativa, y el dominio de conocimientos sobre la profesión, el sistema educativo y las políticas vigentes. 


\section{Orientaciones para la elaboración de marcos para la enseñanza}

A partir del análisis de los cuatros marcos para la enseñanza y la revisión de la literatura respecto al tema, se presentan algunas orientaciones que podrían ser útiles en la elaboración de marcos para la enseñanza o criterios de desempeño profesional docente.

1. Se considera importante sustentar el marco con una teoría acerca de los procesos de enseñanza aprendizaje. Por ejemplo, el marco de Danielson se fundamenta en el enfoque constructivista de la educación (2013b).

2. Un instrumento de este tipo implica una estrategia inclusiva en la que se consulte a diversos actores sobre el contenido y énfasis de los marcos (Meckes, 2013). Entre los grupos que participan en la elaboración de los marcos se encuentran: los ministerios o secretarias de educación y otras instancias públicas de los países, organismos nacionales o internacionales, académicos e investigadores reconocidos en el tema, universidades, colegios y sindicatos de profesores, y los propios docentes, entre otros miembros de la comunidad educativa.

3. Es fundamental elaborar los marcos a partir de la realidad docente. Esto se puede favorecer con la participación de docentes que representen las diversas modalidades y contextos del país o, como en el caso de México, con la observación de videograbaciones de clase de una muestra de escuelas de cada una de las entidades del país (México, Secretaría de Educación Pública, 2010).

4. Es necesario que se definan los elementos de la estructura del marco en el documento de trabajo con la finalidad de facilitar su comprensión a todas las audiencias. Existe una diversidad terminológica con respecto a los componentes de los marcos. Los estándares y competencias se caracterizan por tener diversas definiciones dado que provienen de otras disciplinas y suelen utilizarse diversos términos como sinónimos de competencias y estándares (Barrera y Myers, 2011; Tobón, 2006).

5. Es importante que la finalidad del marco quede claramente establecida en el instrumento y que esta se respete por los tomadores de decisiones. Por ejemplo, si el marco fue diseñado para la evaluación formativa, y se desea utilizar con otros fines que impacten la carrera docente, se tendría que revisar el acuerdo original sostenido con los colectivos afectados. 


\section{Reflexiones finales}

Los marcos de referencia para la docencia y la evaluación de su desempeño son una base para los programas de formación de profesores, así como para el establecimiento de las etapas y requisitos de la trayectoria docente (Vaillant, 2004). Los sistemas revisados evidencian la relación que existe entre el establecimiento de referentes para la formación docente y la evaluación de los profesores, de manera que una vez definidos los marcos de desempeño permitan orientar políticas y programas dirigidos a ambos procesos.

Los fines de la evaluación de la docencia varían de un país a otro. En la mayoría de los casos se propone como un proceso de evaluación formativa que implica la autoevaluación y la reflexión del docente sobre su práctica. A pesar de que la evaluación sumativa no sea un objetivo propio del marco, los sistemas educativos también utilizan estos criterios para realizar procesos de acreditación, la aprobación, la promoción o el incremento salarial de los docentes. No obstante, es muy importante valorar los riesgos que representa la evaluación de alto impacto para los fines formativos.

Es probable que las decisiones respecto a los términos utilizados en los marcos se relacionen más con aspectos de orden político y social que técnico. Por ejemplo, se utilizan otros términos, como criterios o componentes, para referirse a estándares, ya que el concepto es rechazado por algunos actores educativos dado que se asocia con la homogenización de la profesión y práctica docente por su origen en los procesos de estandarización y control del sector industrial (Barrera y Myers, 2011; Meckes, 2013).

A partir de la experiencia de los países que han elaborado criterios para el desempeño de los profesores y las recomendaciones realizadas por los organismos internacionales se identificaron algunas orientaciones para la construcción de marcos para la enseñanza, que constituyen un punto de partida para la discusión y el desarrollo de una propuesta más amplia de referentes para la elaboración de dichos marcos.

\section{Referencias}

Barrera, Iván y Myers, Robert. (2011). Estándares y evaluación docente en México: el estado del debate. Chile: PREAL.

Camero, Mercedes. (2008). La evaluación por competencias, mitos, peligros y desafíos. Educere: Revista Venezolana de Educación, (43), 805-814.

Chile, Gobierno de Chile. (2011). Marco para la Buena Enseñanza. Santiago: Autor. 
Danielson, Charlotte. (2013a). The framework. Estados Unidos: The Danielson Group. Recuperado de http://danielsongroup.org/framework/

Danielson, Charlotte. (2013b). The framework for Teaching. Evaluation Instrument. Estados Unidos: The Danielson Group.

Feldman, Daniel y laies, Gustavo. (2010). Competencias docentes: un marco conceptual para su definición. Recuperado de http://www.fundacioncepp.org.ar/2011/04/competencias-docentes-un-marcoconceptual-para-su-definicion/

Ingvarson, Lawrence y Kleinhenz, Elizabeth. (2006). Estándares profesionales de práctica y su importancia para la enseñanza. Revista de educación, (340), 265-298.

Jornet Meliá, Jesús, Leyva Barajas, Yolanda y Sánchez Delgado, Purificación. (2008). Dimensiones de la clasificación de los procesos de evaluación educativa. En Jesús Miguel Jornet Meliá y Yolanda Edith Leyva Barajas (comps.), Conceptos, metodología y profesionalización en la evaluación educativa 2007 (pp. 67-131). México: Instituto Internacional de Investigación y Tecnología Educativa de México (INITE).

Manzi, Jorge, González, Roberto y Sun, Yulan. (2011). La evaluación docente en Chile. Chile: Facultad de Ciencias Sociales/MIDE UC.

Meckes, Lorena. (2013). Estándares y formación docente inicial. Borrador para discusión. Recuperado de http://www.politicasdocentesalc.com/images/stories/actividades/EST\%C3\%81NDAR ES\%20Y\%20FORMACI\%C3\%93N\%20DOCENTE\%20INICIAL\%20Borrador\%20Lor ena\%20Meckes.pdf

México, Secretaría de Educación Pública. (2010). Estándares de Desempeño Docente en el Aula para la Educación Básica. Borrador para Discusión. México: Autor. Recuperado

de http://asignaturadeartes.files.wordpress.com/2011/11/estandadres desemp doc en el aula de educ basica en mexico esp.pdf

Organización para la Cooperación y el Desarrollo Económico (OCDE). (2010). Specific Policy Recommendations on the Development of a Comprehensive In-Service Teacher Evaluation Framework. Recuperado de http://www.oecd.org/edu/school/48481142.pdf

Oficina Regional de Educación para América Latina y el Caribe, Organización de las Naciones Unidas para la Educación, la Ciencia y la Tecnología. (2013). Antecedentes y Criterios para la Elaboración de Políticas Docentes en América Latina y el Caribe. Santiago: Autor.

Perú, Ministerio de Educación. (2012). Marco de Buen Desempeño Docente. Perú: Autor. 
Tobón, Sergio. (2006). Aspectos básicos de la formación por competencias. Recuperado de

http://maristas.org.mx/gestion/web/doctos/aspectos basicos formacion competenci as.pdf

Vaillant, Denise. (2004). Construcción de la profesión docente en América Latina. Tendencias, temas $y$ debates. PREAL. Recuperado de http://www.oei.es/docentes/articulos/construccion profesion docente AL vaillant.pd $\underline{f}$ 